\title{
Transitive Relationship
}

National Cancer Institute

\section{Source}

National Cancer Institute. Transitive Relationship. NCI Thesaurus. Code C75921.

A binary relation such that whenever an element $a$ is related to an element $b$, and $b$ is in turn related to an element c, then a is also related to c. 